\title{
Thrombotic microangiopathy as first manifestation of acute human immunodeficiency virus infection: a case report and review of the literature
}

\author{
M. Sarmiento ${ }^{1 *}$, M. E. Balcells ${ }^{2}$ and P. Ramirez ${ }^{1}$
}

\begin{abstract}
Background: We present the case of a patient with acute human immunodeficiency virus infection and a thrombotic microangiopathy as the first clinical manifestation, a presentation that has not, to the best of our knowledge, been previously reported.

Case presentation: A 35-year-old Bolivian man presented with epistaxis and thrombocytopenia. We found microangiopathic anemia, lymphopenia, elevated lactate dehydrogenase, progressive acute renal failure, negative direct antiglobulin test, and normal activity of ADAMTS13. An human immunodeficiency virus ELISA test was negative, with an human immunodeficiency virus viral load of 10,000,000 RNA copies/mL. Antiretroviral therapy and three sessions of therapeutic plasma exchange were able to control thrombotic microangiopathy.

Conclusions: Hematologic manifestations of human immunodeficiency virus infection are frequent. However, the debut of acute human immunodeficiency virus infection with thrombotic microangiopathy is a rare event. A high index of suspicion and early treatment is required.
\end{abstract}

Keywords: Human immunodeficiency virus, Acute HIV infection, Thrombotic microangiopathy

\section{Background}

In the majority of cases, early human immunodeficiency virus (HIV) infection is asymptomatic [1]. In those patients with symptoms a variety of clinical manifestations may be seen, including hematological alterations. Anemia and leukopenia can be found in up to $70 \%$ of patients at some point during the course of this viral infection, while thrombocytopenia is usually mild and occurs at the beginning of the infection due to lower production and increased platelet destruction rate [2,3]. Other coagulation disorders such as antiphospholipid syndrome-associated thrombosis and protein $\mathrm{C}$ and protein $\mathrm{S}$ deficiency can also occur, but infrequently. Thrombotic thrombocytopenic purpura (TTP) and thrombotic microangiopathy (TMA) have been occasionally described in patients with

\footnotetext{
* Correspondence: mauriciosarmiento@hotmail.com

${ }^{1}$ Departamento de Hematología y Oncología, Facultad de Medicina, Pontificia Universidad Católica de Chile, Santiago, Región Metropolitana, Chile

Full list of author information is available at the end of the article
}

HIV in advanced stages of infection. However, the debut of acute HIV infection with TTP or TMA is rare [4]. We present a case of a patient with acute HIV infection with a severe TMA and a complete response to therapeutic plasma exchange (TPE) and antiretroviral therapy (ART).

\section{Case presentation}

A 35-year-old previously healthy Bolivian man presented with continuous epistaxis, fever, fatigue, and sore throat. He had a history of unprotected sexual activity during the previous month. A physical examination on admission revealed no major alterations, with the exception of ear, nose, and throat evaluation that showed an intense mucosal bleeding without any visible injury and only partial response to nasal packing. Laboratory tests showed lymphopenia, anemia, severe thrombocytopenia, acute renal failure, lactate dehydrogenase (LDH) twice over normal value, and hyperbilirubinemia that progressively worsened during the first week of hospitalization (Table 1). A specific 
Table 1 Laboratory values at diagnosis and follow-up

\begin{tabular}{llllll}
\hline & Day 1 & Day 4 & Day 8 & Day 15 & Day 90 \\
\hline Hemoglobin $\mathrm{gr} / \mathrm{dL}$ & 11.4 & 9.4 & 11.9 & 10 & 11 \\
Leucocytes $\times 1000$ cells/uL & 5.6 & 3.8 & 6.2 & 5.2 & 5.4 \\
Lymphocytes $\times 1000$ cells/uL & 0.8 & 0.9 & 1.2 & 1.4 & 1.8 \\
Platelets $\times 1000$ cells/uL & 17 & 12 & 90 & 308 & 420 \\
Creatinine $\mathrm{mg} / \mathrm{dL}$ & 1.2 & 1.6 & 0.8 & 0.8 & 0.9 \\
Bilirubin $\mathrm{mg} / \mathrm{dL}$ & 1.33 & 1.9 & 1.2 & 0.8 & 0.7 \\
Lactate dehydrogenase U/L & 600 & 1800 & 457 & 413 & 300 \\
Schistocytes \% & 3 & 4 & 1 & 0 & 0 \\
Fibrinogen $\mathrm{mg} / \mathrm{dL}$ & 325 & 400 & & 218 & \\
ADAMTS13 \% & & & 120 & & \\
Haptoglobin $\mathrm{mg} / \mathrm{dL}$ & 25 & & 80 & 120 & \\
\hline
\end{tabular}

Reference values: hemoglobin 12-16 gr/dL, leucocytes 4.5-11.0/uL, lymphocytes $1.2-4.5 \times 10^{3} / \mathrm{uL}$, platelets $150-400 \times 10^{3} / \mathrm{uL}$, creatinine $0.6-1.1$ $\mathrm{mg} / \mathrm{dL}$, bilirubin $0.6-1.0 \mathrm{mg} / \mathrm{dL}$, lactate dehydrogenase 180-225 U/L, schistocytes $<0.5 \%$, fibrinogen $200-400 \mathrm{mg} / \mathrm{dL}$, ADAMTS13 $>20 \%$, haptoglobin 20-200 mg/dL. ADAMTS13 disintegrin and metalloproteinase with a thrombospondin type 1 motif, member 13

hematological study was conducted and schistocytes were found along with negative direct antiglobulin test (Fig. 1). TTP was suspected and TPE was promptly initiated on the fourth day of admission; he reached normal platelet counts and renal function after three daily TPE procedures. His disintegrin and metalloproteinase with a thrombospondin type 1 motif, member 13 (ADAMTS13) level was normal at day 8 (118\%; normal value 20 to $100 \%)$. Infectious diseases investigations showed a negative ELISA HIV test, but a plasma HIV viral load of $>10$ million/RNA copies per $\mathrm{mL}$ and slightly low CD4 cell count (398 cell/uL). We did not find any opportunistic infections or other viral infections. Acute HIV infection was diagnosed and ART was started with tenofovir, emtricitabine, and raltegravir. After a 13-day

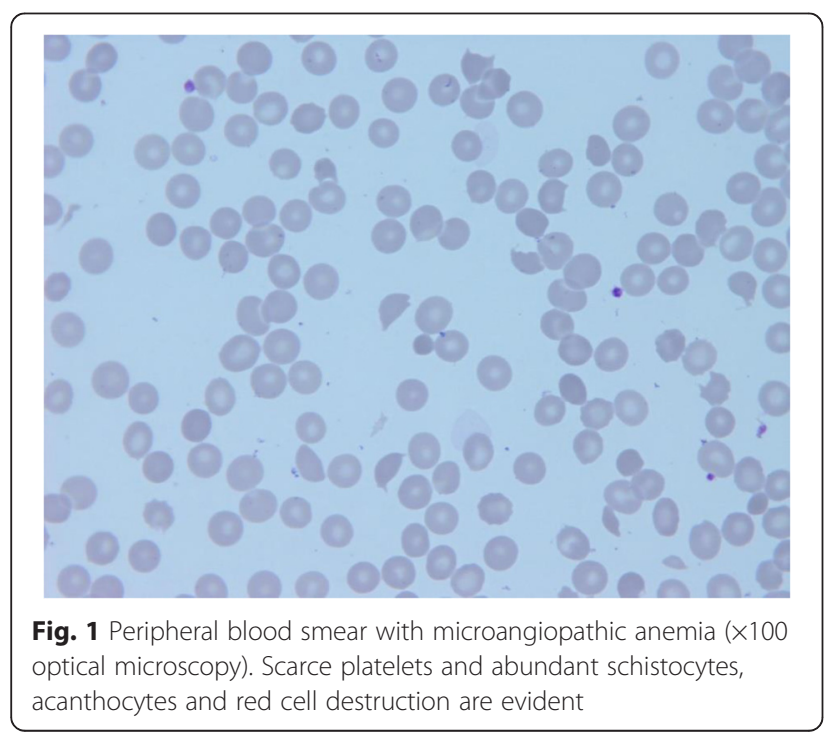

hospital stay, he was discharged in good condition without any further evidence of TMA. A 6-month follow-up showed he had good tolerance to antiretroviral treatment and he had normal blood counts and renal function (Fig. 2).

\section{Discussion}

This case demonstrates the wide variation of clinical manifestations found in patients with early HIV infection. The most frequent hematological findings in this condition are changes to peripheral blood cells, although coagulation disorders may also occur. Furthermore, the coexistence of immunological-mediated thrombocytopenia with TTP has been reported in chronic HIV infection [5].

Thrombotic manifestations such as serious thrombosis, TTP, and TMA usually occur in late stages of chronic HIV infection or in patients with poor adherence to ART [6]. The classic form of TTP is caused by an acquired or hereditary malfunction/deficit of ADAMTS13, which fails to cleave the ultra-large multimers of the von-Willebrand factor (v-WF) and produces classic thrombotic microangiopathic anemia and multiorgan failure [7]. On the other hand, TMA associated with drugs, neoplasia, or infections has the same features as classic TTP but other mechanisms lead to thrombosis without ADAMTS13 inhibition. In HIV

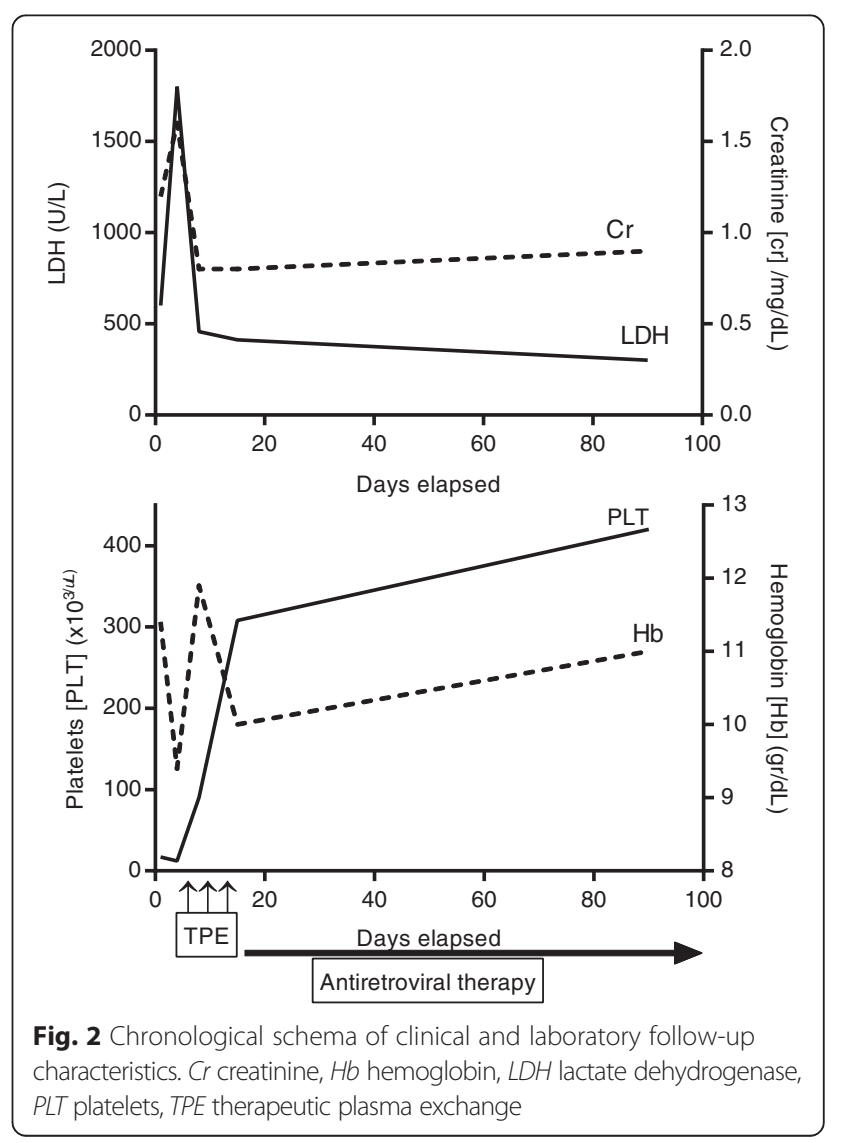


infection, multiple alterations have been described that can induce either immune TTP triggered by dysfunctional ADAMTS13 or TMA generated by diverse conditions such as alterations to complement proteins, endothelial injury secondary to cytokines induced by the virus, or endothelial cell damage directly mediated by viral particles [8]. Because of these multiple pathological pathways, treatment of patients with either TTP or TMA associated to HIV should be directed to rapidly control the viral load, reduce the virus-induced immunosuppression, and replace the defective ADAMTS13 and coagulation proteins by TPE.

Miller et al. showed that $12 \%$ of patients diagnosed with TTP had concomitant HIV infection, and they were more often found to be at advanced stages of the disease with profound immunosuppression. In this situation, there was a clear therapeutic benefit of adding ART in addition to TPE [9]. However, in the largest cohort of patients, the Oklahoma Thrombotic Thrombocytopenic Purpura - Hemolytic Uremic Syndrome (TTP-HUS) register, only $1.84 \%$ of 326 patients with TTP had HIV infection and the authors concluded that HIV infection, similar to other inflammatory conditions, could trigger acute episodes of TTP in susceptible patients. Moreover, HIV-induced oncological and infectious disorders could mimic the clinical features of TTP and must be included in the differential diagnosis [10].

Our patient's case is remarkable in some aspects. The first and perhaps most interesting is that his acute HIV infection debuted with severe TMA, which to the best of our knowledge has not been previously reported. Negative anti-HIV antibodies with a very high HIV viral load defines acute HIV infection and is characteristically associated with extremely high viremia. Our patient emphasized that sexual risk behaviors were recent events $(<1$ month), and that bleeding and constitutional symptoms appeared almost immediately upon presumed HIV exposure.

In this case, TMA was quickly controlled with TPE and prompt ART initiation. Although TPE could have had some role in our patient's recovery, information is not available to support use in TMA. In this particular case, the use of TPE was an extreme action given the severity of the patient's symptoms. In clinical hematology practice it is well recognized that TPE is mainly beneficial in cases where TTP coexists with an immune inhibitor of ADAMTS13, and not in non-immune TMA forms. However, there are several recent reports showing that TPE and other immunological therapies such as rituximab and eculizumab may be useful in cases not necessarily associated with autoimmunity. This effect could be associated to reposition of other coagulation regulatory proteins or modulation of this effect could be associatedwith reposition of other coagulation regulatory proteins or B lymphocytes modulation [11].

\section{Conclusions}

We presented an unusual manifestation of acute HIV infection associated with TMA that responded successfully to TPE and prompt start of ART. In patients with TTP or TMA, HIV testing must be mandatory. Furthermore, in patients with known HIV infection, physicians should be aware of the probability of development of thrombotic complications including TTP and TMA.

\section{Acknowledgments \\ The authors declare that they have no acknowledgements in this manuscript.}

\section{Authors' contributions}

MS, MEB, and PR contributed equally in the treatment of the patient and writing of manuscript. All authors read and approved the final manuscript.

\section{Competing interests}

The authors declare that they have no competing interests.

\section{Consent}

Written informed consent was obtained from the patient for publication of this case report and accompanying images. A copy of the written consent is available for review by the Editor-in-Chief of this journal.

\section{Author details}

${ }^{1}$ Departamento de Hematología y Oncología, Facultad de Medicina, Pontificia Universidad Católica de Chile, Santiago, Región Metropolitana, Chile. ${ }^{2}$ Departamento de Enfermedades Infecciosas, Facultad de Medicina, Pontificia Universidad Católica de Chile, Santiago, Región Metropolitana, Chile.

Received: 11 September 2015 Accepted: 6 May 2016

Published online: 07 June 2016

\section{References}

1. Rosenberg NE, Pilcher CD, Busch MP, Cohen MS. How can we better identify early HIV infections? Curr Opin HIV AIDS. 2015;10(1):61-8.

2. Hicks CB. Duke-Unc Acute HIV Infection Consortium. AIDS Res Hum Retroviruses. 2013;29(1):121.

3. Zucker-Franklin D, Cao YZ. Megakaryocytes of human immunodeficiency virus-infected individuals express viral RNA. Proc Natl Acad Sci U S A. 1989; 86(14):5595.

4. Becker S, Fusco G, Fusco J, Balu R, Gangjee S, Brennan C, et al. HIV-associated thrombotic microangiopathy in the era of highly active antiretroviral therapy: an observational study. Clin Infect Dis. 2004;39 Suppl 5:S267.

5. Routy JP, Beaulieu R, Monte M, Saint-Louis J, Sauvageau G, Toma E. Immunologic thrombocytopenia followed by thrombotic thrombocytopenic purpura in two HIV1 patients. Am J Hematol. 1991;38(4):327-8.

6. Rakhmania N, Wong E, Davies J, Ray P. Hemorrhagic stroke in an adolescent female with HIV-associated thrombotic thrombocytopenic purpura. AIDS Clin Res. 2014:5(6):311.

7. Furlan M, Robles R, Galbusera M, Remuzzi G, Kyrle PA, et al. von Willebrand factor-cleaving protease in thrombotic thrombocytopenic purpura and the hemolytic-uremic syndrome. N Engl J Med. 1998;339:1578-84.

8. Brecher ME, Hay SN, Park YA. Is it HIV TTP or HIV-associated thrombotic microangiopathy? J Clin Apher. 2008;23:186-90.

9. Miller RF, Scully M, Cohen H, Roedling S, Starke R, et al. Thrombotic thrombocytopaenic purpura in HIV-infected patients. Int J STD AIDS. 2005; 16:538-42.

10. Benjamin M, Terrell DR, Vesely SK, Voskuhl GW, Dezube BJ, Kremer Hovinga $J A$, et al. Frequency and significance of HIV infection among patients diagnosed with thrombotic thrombocytopenic purpura. Clin Infect Dis. 2009:48(8):1129-37.

11. Murugapandian S, Bijin B, Mansour I, Daheshpour S, Pillai BG, Thajudeen B, et al. Improvement in gemcitabine-induced thrombotic microangiopathy with rituximab in a patient with ovarian cancer: mechanistic considerations. Case Rep Nephrol Dial. 2015;5(2):160-7. 